\title{
DNA: An Investigation of the Three-Day Strength Guarantee Made by Magnum Nutraceuticals in Regards to their Branch Chained Amino Acid Supplement DNA Nicholas Conley ${ }^{\mathrm{a}}$
}

This small pilot study used a double-blinded, between subject experimental design in an attempt to investigate the claim made my Magnum Nutraceuticals in regards to their newest supplement, DNA. The manufacturer has a three-day guarantee saying that if an individual takes this supplement for three days then they will experience an increase in strength (more repetitions to their normal weight lifting regiment). Two groups (placebo and experimental) performed three different weight lifting exercises (squats, chin-ups, bench press) while lifting $1 / 2$ of their body weight per manufacturer's instructions. The groups met on two different occasions separated by three days in between. The first meeting the participants completed the three aforementioned exercises and were assigned into the placebo or intervention group. After the baseline the participants were instructed to take their pills as directed for the next three days with the third day being a repeat of the three exercises to compare with baseline. Once all 13 participants ( $\mathrm{N}=7$ intervention, $\mathrm{N}=6$ placebo) completed the final workout, the results were scored and significant differences were noted in all three exercises after excluding the numbers from the outlier in the experimental group. There was one minor adverse effect (dyspepsia) experienced with the use of the supplement though all ingredients contained within DNA had GRAS status as designated by the FDA. It was concluded that participants taking DNA had a significant increase in strength when compared against the placebo group for squats, bench, and pull-ups. DNA was shown to be reasonably safe and may help athletes increase repetitions when training with a similar exercise regimen.

Keywords: BCAA, strength supplements, supplemental nutrition

\section{Introduction}

Magnum Nutraceuticals is a supplement company based in the Canada area and has been in business since 2005. Magnum has been a popular brand among athletes using supplementation as evident by the fact that the company's products can be found in over 40 different countries. The supplement market is a multimillion-dollar industry where many different companies attempt to distinguish their products with general claims and guarantees. Magnum attempts to distinguish itself by commissioning certified good manufacturing practices (CGMP), site licensed pharmaceutical facilities that undergo regular government auditing for quality assurance, and the use of pharmaceutical grade ingredients.

As competition in the supplement market increases so does the pressure for supplement companies to support the general claims they make on selected products. Magnum Nutraceuticals manufacturers a branched-chained amino acid supplement, which they label as DNA with the claim of a three-day strength guarantee.

The premise behind this claim lays in the fact that protein supplements have always been a popular choice for athletes because protein is one of the three macronutrients (carbohydrates and fats being the other two) used by the body to support essential biological and provide bulk energy ${ }^{4}$. Proteins begin digestion into amino acids in the stomach and then are built into new proteins as needed by the body.

Amino acids are the basic building blocks of proteins and serve a variety of functions within the body ${ }^{7}$. Amino acids can be classified as essential or non-essential (those obtained through the diet). There are three amino acids further classified as branched chain amino acids (BCAA's) because of their chemical structure and include leucine, isoleucine, and valine ${ }^{3}$. These are the three most known among athletes because they are used in the muscles. It is suggested that a high intake of BCAA's will stimulate amino acids to be used for building new proteins (thus increase muscle mass) instead of being burned as energy ${ }^{8}$. BCAA's are a common product produced by supplement companies that focus advertising efforts to consumers looking to increase strength.

There has been a great deal of research surrounding BCAA's as a result. Some of these speak favorably towards supplement use with a 2013 study concluding that a BCAA pre-workout supplement suggested an increase in lean body mass and muscle hypertrophy in resistance-trained men ${ }^{\mathbf{1 4}}$. Other such studies have even suggested other applications for BCAA products by supplementing to reduce muscle soreness and damage ${ }^{\mathbf{1 6}}$. Favorable results however aren't widely accepted as research has also suggested no such correlation exist. In 2014 a study suggested that BCAA supplementation was ineffective to prevent muscle power loss or damage during exercise1 and a 2011 suggest clinical efficacy regarding similar supplements are simply not strong enough to make recommendations on ${ }^{\mathbf{1 0}}$. These conflicting reports make the case for further research to accurately portray the role of BCAA supplementation in athletes.

Despite these varied findings, Magnum Nutraceuticals has recognized the popularity of branch-chained amino acids and associated a three-day strength guarantee on their BCAA supplement, DNA. The purpose of this research is to investigate the three-day guarantee and provide consumers with results so that they can make an educated decision when it comes to selecting a supplement. The ingredients of DNA all fall within the Food Drug Administration's GRAS (generally regarded as safe) index because of their high tolerability and safe profile. The manufacturer (Magnum Nutraceuticals) has provided a general overview of the product as seen in Table $\mathbf{7}$ as well as an ingredient list with proposed mechanism of actions for each in Table 8 
Table 7: Manufacturer Product Overview

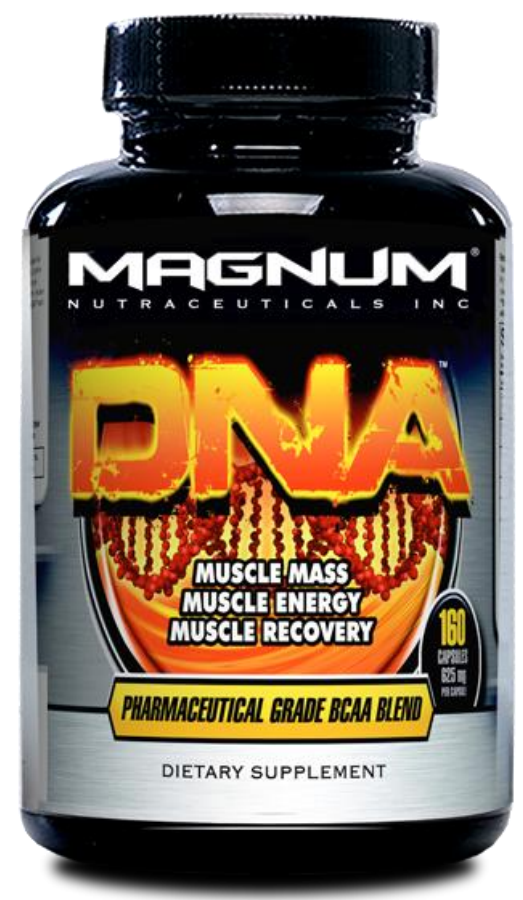

\section{What is Magnum DNA®?}

Magnum DNA $®$ is an anabolic formula that combines the powerful muscle-regenerating effects of Branched Chain Amino Acids (BCAAs) with the hardening and muscle expanding Glycine/Arginine ingredient. DNA ${ }^{\circledR}$ also contains the intense anabolism-triggering ingredient L-Leucine Alpha KIC to ensure the most complete, natural muscle gains rarely produced in a nonhormonal supplement. These scientifically proven ingredients have been known to improve density of muscles and strength, recovery and athletic performance. Only a formula this concentrated comes with a 3-day strength guarantee!* Branched Chain Amino Acids (BCAAs) are known as the building blocks of muscle. They must be present for muscle development and growth. The essential BCAAs are leucine, isoleucine and valine. These BCAAs are classified as "essential" because the human body does not produce them - they must be taken through supplementation.

Magnum DNA $®$ produced the following results

- Instant strength • Improved recovery $\bullet$ Increased endurance • Increased protein synthesis (utilization of protein) $\bullet$ Long-term increased lean muscle mass $\bullet$ Long-term muscle hardening and definition

What is unique about Magnum DNA®?

- It offers 7 of the most innovative, pure, Pharmaceutical Grade sources of BCAAs, including the latest Ethyl Ester \&

Ketoisocaproate Calcium technology • Ethyl Ester is the first bonding molecule to pass the blood-brain barrier, thus effectively saturating every cell in the body with a larger dose of the attached BCAA - Ketoisocaproate Calcium (KIC) is perfectly anti-catabolic - it assists in muscle growth by changing the environment in your body from catabolic (muscle wasting) to anabolic (muscle building) $\bullet$ It also supplies a potent dose of Glycine-L-Arginine - an ingredient scientifically proven to enhance muscle performance and recovery from fatigue by sustaining muscle force and overall output Manufacturer Product Overview

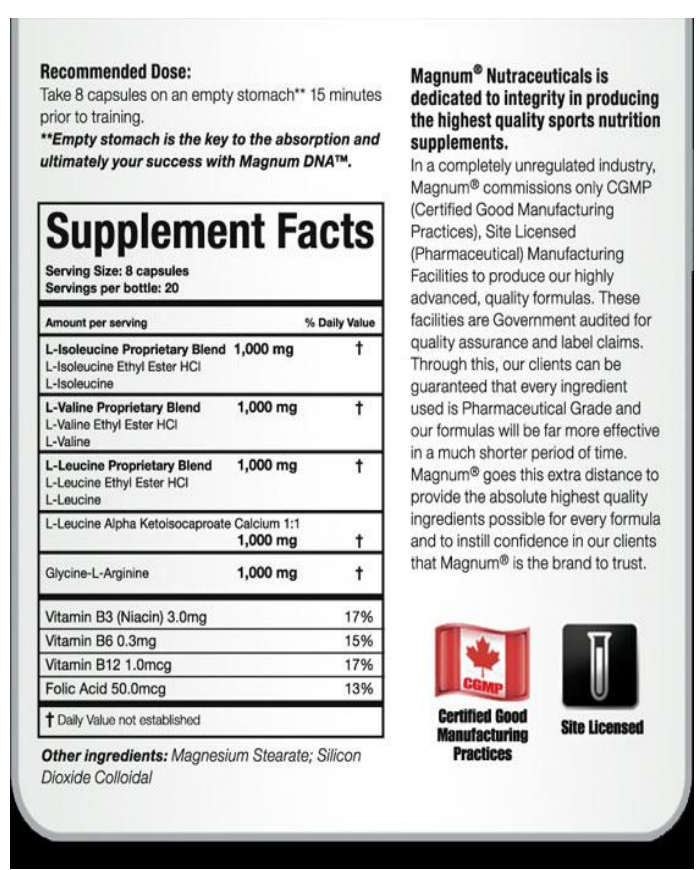

\section{Who is Magnum DNA® meant for?}

Uses weights with their training program - Is looking to improve strength and endurance $\cdot$ Is trying to increase lean muscle mass \& improve body composition • Is a bodybuilder, fitness competitor or athlete

\section{The World's 1st Pharmaceutical Grade Anabolic Activating Experience!}

An extremely anabolic pre-workout formula unlike any other! DNA users routinely report that they are able to do more reps with the same weight from their previous workout from their very first dose! Strength, power, recovery, endurance $\&$ muscle hardening all attained within 3 days! DNA is truly an awesome supplement because it's the only BCAA based anabolic formula that you will feel from your very first workout.

\section{How to use}

Serving Size 8 capsules 160 capsules per bottle absorption and ultimately your success with Magnum DNA 


\section{Table 8: Ingredient Profile}

The ingredients contained in DNA are known as branch chained amino acids (BCAAs) and are readily obtained through a protein rich diet. The low likelihood of adverse reactions from intake of BCAAs as well as their safe profile has earned the ingredients contained within DNA generally regarded as safe (GRAS status) from the FDA. This makes for a very tolerable product whose potential pharmaceutical nature is outlined below by Magnum Nutraceuticals. This information should give consumers a better understand at the proposed science behind the supplement.

Magnum's DNA Ingredient Profile - Serving Size: 8 capsules

Recommended Dose: Take 8 capsules on an empty stomach 15 to 20 minutes prior to training

\section{BCAA's In General}

These three amino acids - Valine, Isoleucine, and Leucine - account for one third of the protein in muscle tissue. This significant contribution makes them important for helping build muscle and increase energy in muscle cells. They are also converted to other amino acids when deficiencies arise.

\section{1) L-Isoleucine Proprietary Blend 1,000mg (L-Isoleucine Ethyl Ester HCI, L-Isoleucine)}

Isoleucine is an essential branched-chain amino acid (BCAA). A building block of protein, isoleucine plays a vital role in protein synthesis, muscle building, and preventing muscle loss.

Isoleucine, like all BCAA's, is used by athletes whose muscles are excessively stressed by overtraining to promote healing of injured tissues, speed recovery, protect against muscle-tissue breakdown, and possibly increase lean mass. Isoleucine also stabilizes blood sugar to regulate energy levels during exercise and is needed for the formation of hemoglobin, which carries iron in the blood. In addition, Isoleucine is used as fuel by muscle cells and may spare other amino acids from being burned.

\section{2) L-Valine Proprietary Blend 1,0000mg (L-Valine Ethyl Ester HCI, L-Valine)}

Valine, an essential branched-chain amino acid (BCAA), is an energy source for muscles and helps repair tissues. A building block of protein, Valine also plays a vital role in muscle building, immune-system function, and balancing natural levels of water and nitrogen. Valine also supplies exercising muscles with fuel, sparing other amino acids and is a unique essential amino acid in that it may act as a stimulant and is stored largely in muscle tissue - where it is used as an energy source when our muscles are activated.

\section{Ingredient Profile}

3) L-Leucine Proprietary Blend 1,000mg (L-Leucine Ethyl Ester HCI, L-Leucine)

\section{L-Leucine Alpha Ketoisocaproate Calcium 1:1 - 1,000mg}

Leucine is an essential branched-chain amino acid (BCAA) used as an energy source for muscles. A building block of protein, Leucine plays a vital role in immune-system function as well as muscle protection, fuel, and repair. Leucine boosts gains in muscle mass by increasing available fuel for muscle cells and preserving muscle energy stores since our muscles use Leucine directly for fueling any work they perform. Researchers estimate that up to $90 \%$ of dietary Leucine is used for energy during exercise and consider it the limiting amino acid when athletes don't consume additional amounts to make up for what's used by the body. With the addition of Alpha-Ketoisocaproic acid ( $\alpha$-KIC) to further enhance the buffering of lactic acid (muscle toxin) and increase the levels of L-Leucine at the site of muscle, DNA's proprietary Leucine formulation aims to increase strength gains and cellular energy beyond all other BCAA formulations.

\section{4) Glycine-L-Arginine - 1,000mg}

When Arginine is combined with Glycine, it's been shown to increase muscle Creatine content - boosting performance during anaerobic training sessions (like weight training), and enhancing muscle and strength capacity. It also increases cellular energy by controlling blood sugar levels, protecting against ATP depletion, and by shuttling toxic substances such as lactic acid out of the body. The combination of these two ingredients has been clinically proven to be superior (versus either ingredient separately) at improving muscular growth before and after trauma, and increasing nitrogen retention following trauma. Further evidence has shows that during rapid growth phases (such as weight training), the body demands even more Glycine and some studies have indicated that Glycine causes an increase in strength, perhaps attributable to its effects on growth-hormone levels and/or cell volume.

\section{5) Vitamin B3 (Niacin) - 3.0mg}

$\mathrm{B} 3$ or niacin plays a key role in over 100 functions necessary for health and is especially important for energy production and, as a vasodilator- increasing blood circulation. It aids in the production of enzymes that convert carbohydrates, glucose, and fats into useable energy and regulates blood glucose levels and supports the body's response to insulin

6) Vitamin B6 - 0.3mg

Vitamin B6 plays a key role in converting the proteins you eat into the amino acids that make up your muscles. In addition, it assists in the availability of energy and the formation of important neurotransmitters (like serotonin) and the maintenance of a healthy immune system. It is a powerful precursor to positive physical and mental well being.

\section{Ingredient Profile}

\section{7) Vitamin B12 - 1.0mcg}

Vitamin B12 is one of the most intriguing vitamins and also one of the most complex. It plays many critical roles in our bodies, including maintenance of our nervous systems, formation of red blood cells, energy metabolism, and the proper functioning of our brains. Its importance to our bodies' optimal performance defines the meaning of the word "essential."

Vitamin B12 has the capacity to increase energy, and a deficiency of B-12 will have a debilitating effect on performance and energy. While serious deficiencies are not a common occurrence in normally healthy people, an athlete's body is taxed by intense workouts and dietary modifications that remove them from the average/normal population. 


\section{8) Folic Acid - 50.0mcg}

Folic acid or folate, one of the B-complex vitamins, is necessary for the synthesis of DNA and SAMe — which keep our cells healthy and able to replicate, as well as enhance mood. It is also necessary to keep homeocysteine levels in check, which is needed for our bodies' metabolism of both amino acids and sugars.

Deficiencies are common and can lead to a wide range of symptoms, including fatigue, depression, and even acne. Plus, it's necessary for the metabolism of amino acids, the building blocks of protein, and to transform sugars into useable energy. It's important to stave off any deficiency of folic acid, as lacking enough of this vitamin can inhibit both muscular and nervous system function.

\section{Methods}

\section{Participants}

13 male participants were recruited for this study. 7 were allocated to the experimental group and 6 were allocated to the placebo group. Each participant was between the ages of 18-44 in the surrounding area of Kernersville, North Carolina. The average age was 28 for the placebo group and 29 for the intervention group. The participants were obtained from a series of flyers that were posted at local gyms. To be considered, selected participants had to be involved in at least one sport regularly (3x a week). Exclusion criteria were comprised of any history of significant injury or medical issues that would prevent the participant from taking part in the selected exercises. The participants were informed that this research was being used to investigate the 3-day strength guarantee of DNA as manufactured by Magnum Nutraceuticals. All personal information regarding the participants was kept confidential and destroyed at the conclusion of the study.

\section{Design}

This study used a double-blinded, between subject experimental design. The independent variable consisted of the participant receiving the intervention (DNA) or the placebo. Three dependent variables were measured: (1) increase in squat repetitions, (2) increase in chin-ups, and (3) increase in bench press repetitions. All exercises were performed at $1 / 2$ the participant's body weight (except for the chin-ups). The placebo group $(\mathrm{N}=6)$ combined with the experimental group $(\mathrm{N}=7)$ gave a total number of 13 participants involved. The participants performed the exercises on the first session that served as their baseline and then were instructed to take the placebo/intervention for three consecutive days with 10 fluid ounces of water. On the third day of taking the supplement/placebo the participants returned to repeat the three exercises. Compliance was self-reported by participants. This was a very small sample size and will serve as a pilot study for future investigations. The Wingate University research review board gave this study an exemption due to minimal risk from ingredients (GRAS Status) and the lack of patient identifying information used. An overview of the design has been illustrated in Table 5.

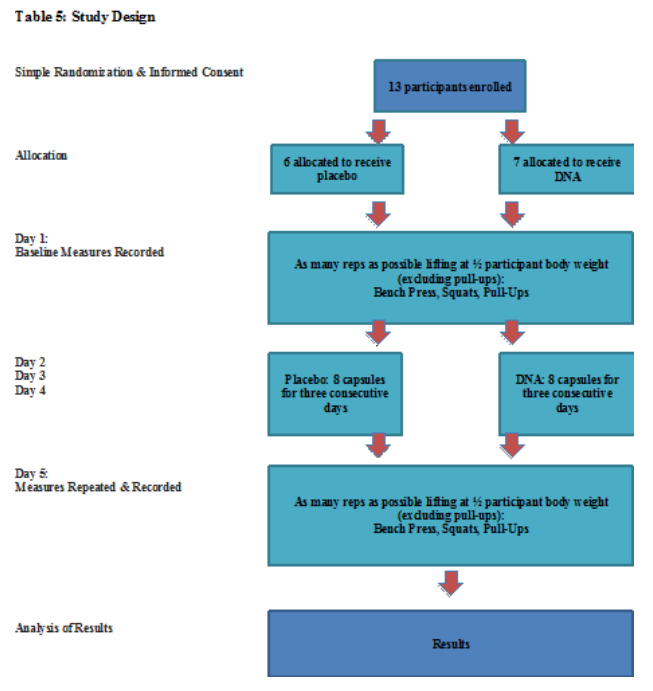

Materials

The supplement DNA and the Placebo were both provided by Magnum Nutraceuticals. The serving size for each was eight capsules to be taken with 10 fluid ounces of water as instructed from the manufacturer. The participant had to take the placebo/intervention for three consecutive days around the same time every day. A brief handout was developed by the researcher to help collect the opinions of the participants, to help assess compliance rates, and to record all repetitions related to the exercises. This handout can be seen in Table 6.

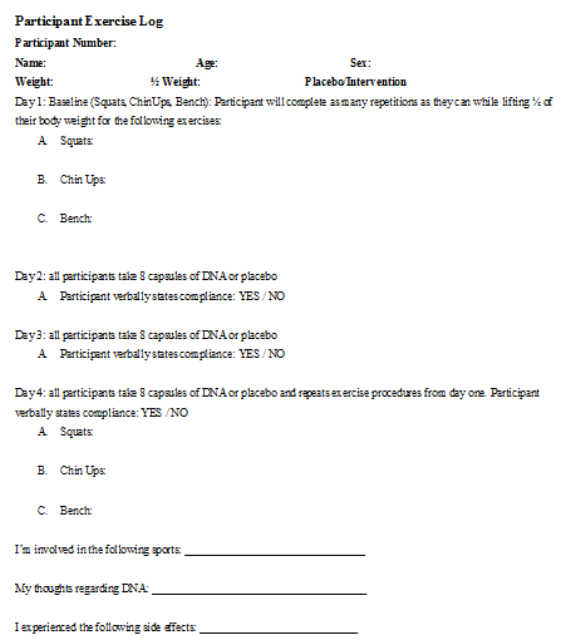

Procedures 
After informed consent was received from all 13 participants, they were categorized into groups using simple randomization. All participants were instructed that they would meet as a group with the researcher on two different occasions that were separated by two days. The participants were asked to maintain normal daily activity while taking part in the study. There were three different measures of the study; (1) increase in squat repetitions, (2) increase in chin-ups repetitions, (3) and an increase in bench press repetitions. A professional bodybuilder demonstrated all exercises to participants before the study. These three exercises were determined by the manufacturer to test the claim that their supplement product DNA will increase strength after three days of consecutive use. A digital scale was used to capture the weight of each participant to determine the amount of weight they would use for each exercise. All exercises were weighted at $1 / 2$ the body weight of each participant (minus chin-ups) as instructed by Magnum Nutraceuticals.

After all three exercises were completed and documented the participants were instructed to take either the placebo/intervention for three days and return to the study on the third day to repeat the exercises. Participants were instructed to continue their daily routines and supplement use. Patients were individually asked to report compliance on the third day. A doctorate student from Wingate University School of Pharmacy conducted the individual sessions of the participants with the supervision of a professional bodybuilder to ensure proper form and technique. The trained student was blinded and individually led each group of participants to utilize a double blind design.

Statistically analysis

An independent t-test (2-tailed) with assumed equal variances was used to conclude if there was a significant difference between the primary endpoints of the intervention and the placebo group. This t-test was implemented three different times for each of the three primary endpoints and then a fourth time with all endpoints classified together. An alpha 0.05 was set to determine significance between each variable. Equal variances were assumed due to the similarities between groups. The outlier for each exercise in the experimental group was identified and excluded to give a total of six numbers from each group. All numbers were calculated as a form of percent increase/decrease in order to compare groups and all half numbers were rounded down.

\section{Results}

Statistical analysis was performed to conclude if the increase in strength from all three measures was due to the intervention. This can be seen in Table 4 while the data collected throughout the study can be seen in Table $\mathbf{1}$ for the experimental group and Table 2 for the placebo group.

In regards to squats $p=<0.05(0.0175)$ thus it was concluded statistically significant. All participants in the experimental group had an increase in repetitions when compared to the placebo group. The outlier from the experimental group during squats was participant \#6. Participant \#6 performed 56 squats on the first day followed by 117 squats on the final day. This resulted in a $108 \%$ percent increase and thus this number will not be included for statistical analysis. This number was the outlier and was ultimately removed because it does not reflect typical results for the average individual and may inflate the overall data resulting in false conclusions. To show a significant difference when the outlier is removed should further strengthen any results concluded from the study.

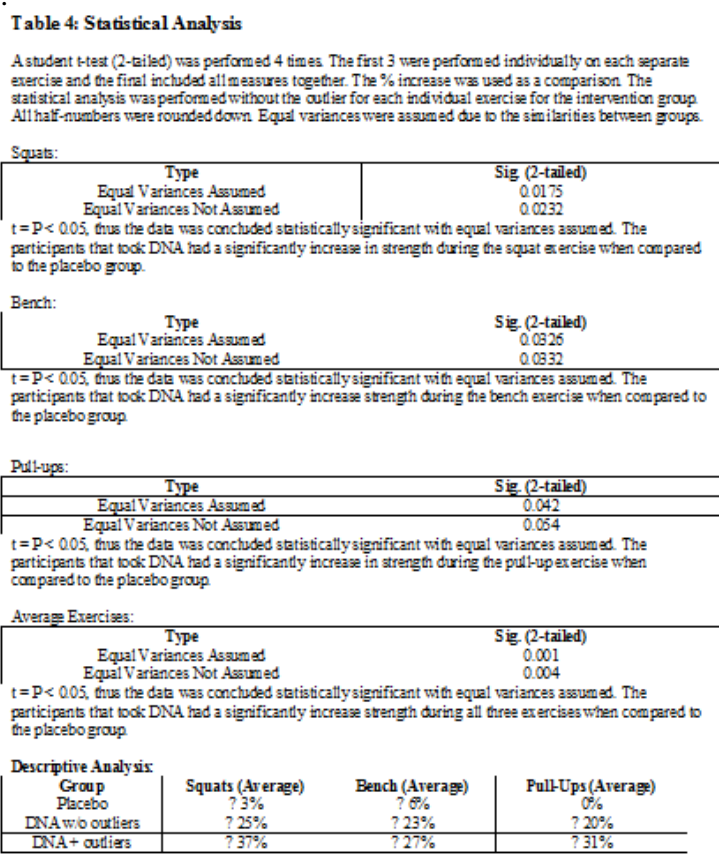

Table 1: Experimental Group Results

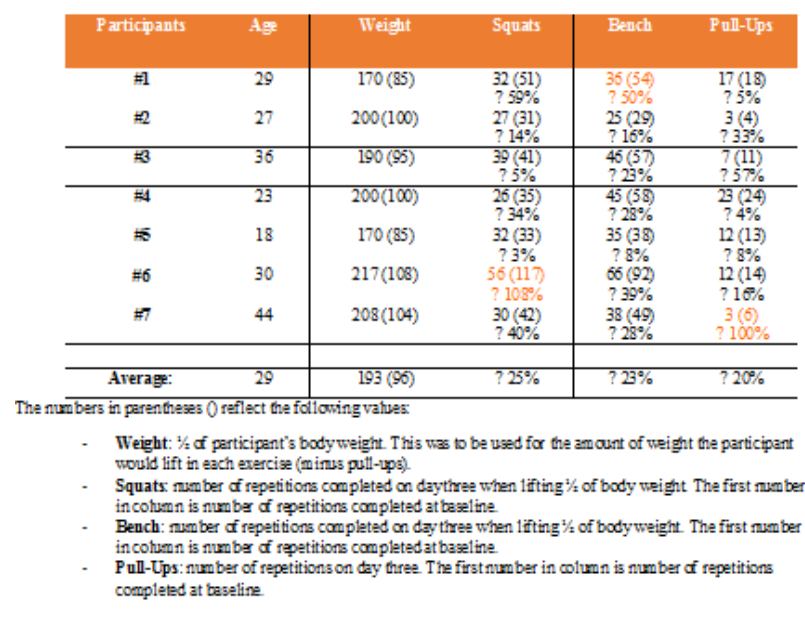

Disaussion:

All participants had $n$ increase in repetitions when compared to baseline in all trees exercises The $\%$ increase can be seen as noted by the ? symbol

fions noted in orang mark outliers and zores will not be used for statistical analysis 


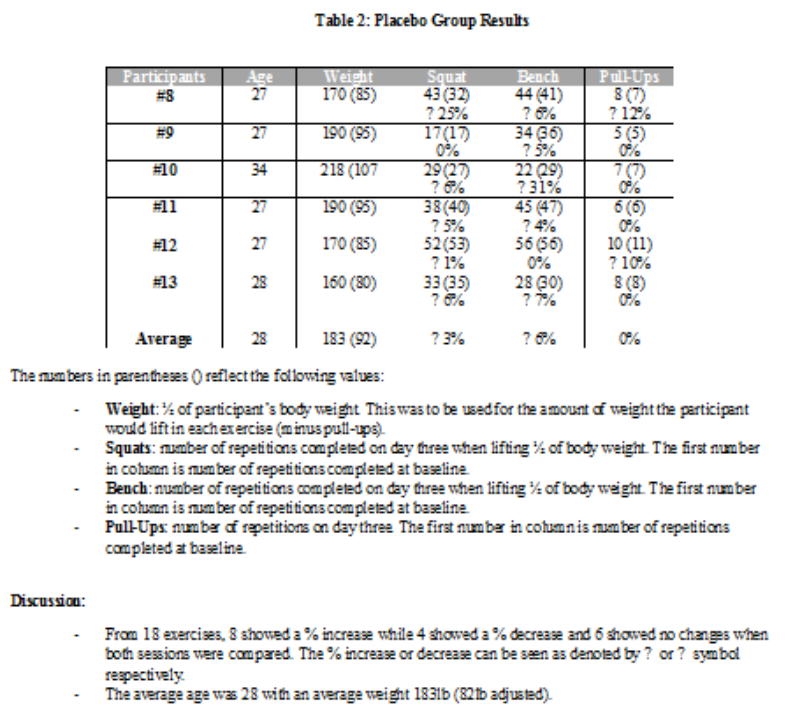

In regards to the bench exercise $p=<0.05(0.0326)$ thus it was concluded statistically significant. All participants in the experimental group had an increase in repetitions when compared to the placebo group. The outlier from the experimental group during bench was participant \#1. Participant \#1 performed 36 repetitions on the first day followed by 54 on the final day. This resulted in a $50 \%$ percent increase and thus this number will not be included for statistical analysis. This number was the outlier and was ultimately removed because it does not reflect typical results for the average individual and may inflate the overall data resulting in false conclusions. To show a significant difference when the outlier is removed should further strengthen any results concluded from the study.

In regards to pull-ups $\mathrm{p}=<0.05(0.042)$ thus it was concluded statistically significant. All participants in the experimental group had an increase in repetitions when compared to the placebo group. The outlier from the experimental group during pull-ups was participant \#7. Participant \#7 performed 3 pull-ups on the first day followed by 6 on the final. This resulted in a 100\% percent increase and thus this number will not be included for statistical analysis. This number was the outlier and was ultimately removed because it does not reflect typical results for the average individual and may inflate the overall data resulting in false conclusions. To show a significant difference when the outlier is removed should further strengthen any results concluded from the study.

The placebo group was involved in 18 individual exercises with 8 showing a $\%$ increase, 4 showing a \% decrease, and 6 showing no change when both sessions were compared.

5 out of the 7 participants in the experimental group were involved in weightlifting compared to 4 out of the 6 in the placebo group. Participant \#2 of the experimental group experienced one minor episode of dyspepsia during the study Participant \#4 reported a bad aftertaste. Self reported data for both groups is seen in Table 3 .

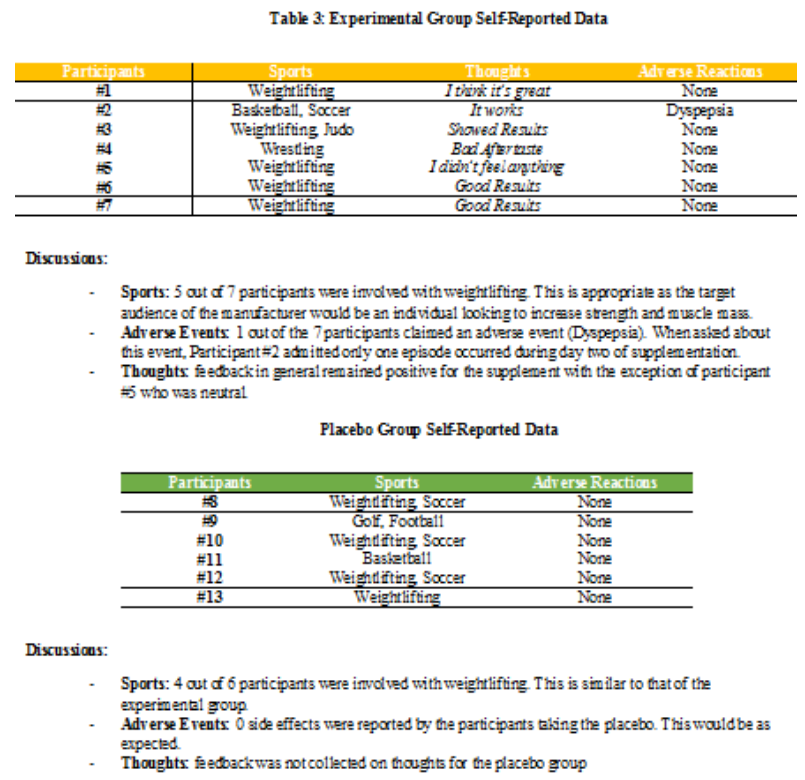

When all exercises were grouped as one $\mathrm{P}<0.05$ (0.001). This concluded that overall the experimental group had a significant increase of strength when comparing the exercises as a percent of increase when compared to the placebo group. The three exercises were also descriptively analyzed as an average. The placebo group had an overall average of percent increase of $-3 \%$ (squats), $6 \%$ (bench), and $0 \%$ pull-ups. In contrast the experimental group had an overall average of percent increase of $25 \%$ (squats), $23 \%$ (bench), and $20 \%$ (pull-ups).

\section{Discussion}

The purpose of this research was to examine the claim from Magnum Nutraceuticals in regards to their branchedchain amino acid supplement DNA and investigate the labeled three-day strength guarantee. At the conclusion of the study it was found that all three measures had a significant increase of repetitions when comparing the intervention group to the placebo group. This would suggest that DNA could possibly show an increase in strength when used for three consecutive days while performing the three selected exercises used in the study.

It is important to note that this study used a small sample size and should serve as the foundation for future studies. The results should not be generalized to the public and it is reminded that all supplements work differently in every individual. This research should provide consumers with some insight regarding the supplement DNA, but it is also advised to consult your physician before partaking in any supplementation or workout regiment. Future studies should replicate the design with the selected exercises within a larger population to see if results can be duplicated. In addition to the small sample size other limitations were the self-reporting of compliance by participants as well as not being able to regulate any other possible supplements used by participants. This could account for the significant increase of strength in the experimental group if participants were using additional supplements that supported muscle development. 
Overall, DNA appears to be a reasonably safe supplement with a tolerable and safe ingredient profile. Previous studies have shown mixed results regarding BCAA's, but some do favorably associate the supplement with increase strength and performance in athletes during exercise training. Research on this supplement should continue to see if further association can be determined, but this study suggests that the three-day strength guarantee held intact for the participants who participated in the three selected exercises.

\section{References}

1) Areces F, Salinero JJ, Abian-Vicen J, Gonzalez-Millian C, Gallo-Salazar C, et al. A 7-day oral supplementation with branched-chain amino acids was ineffective to prevent muscle damage during a marathon. Amino Acids. 2014.

2) Bassit RA, Sawada LA, Bacurau RF, Navarro F, Costa Rosa LF. The effect of BCAA supplementation upon the immune response of triathletes. Medicine and Science in Sports and Exercise. 2000; 32(7):1214-9.

3) Chapter 1. Amino Acids and Proteins. In: Janson LW, Tischler ME. eds. The Big Picture: Medical Biochemistry. New York: McGraw-Hill; 2012.

4) Chapter 1. General Principles \& Energy Production in Medical Physiology. In: Barrett KE, Boitano S, Barman SM, Brooks HL. eds. Ganong's Review of Medical Physiology, 24e. New York: McGraw-Hill; 2012.

5) Chapter 1. Amino Acids and Proteins. In: Janson LW, Tischler ME. eds. The Big Picture: Medical Biochemistry. New York: McGraw-Hill; 2012.

6) Chapter 15. Carbohydrate, Protein, and Water-Soluble Vitamin Assimilation. In: Barrett KE. eds. Gastrointestinal Physiology, 2e. New York: McGraw-Hill; 2014.

7) Chapter 26. Digestion, Absorption, \& Nutritional Principles. In: Barrett KE, Boitano S, Barman SM, Brooks HL. eds. Ganong's Review of Medical Physiology, 24e. New York: McGraw-Hill; 2012.

8) Churchward-Venne TA, Breen L, Di Donato DM, Hector AJ, Mitchell CJ, et al. Leucine supplementation of a lowprotein mixed macronutrient beverage enhances myofibrillar protein synthesis in young men: a double-blind, randomized trial. Americanl Journal of Clinical Nutrition. 2014; 99(2):276-86.

9) Colditz GA. Healthy diet in adults. In: UpToDate, Post TW (Ed), UpToDate, Waltham, MA. (Accessed on February $10^{\text {th }}$, 2014.)

10) Graf S, Egert S Heer M. Effects on whey protein supplements on metabolism: evidence from human intervention studies. Current Opinion in clinical Nutrition and Metabolic Care. 2011; 14(6):569-80.
11) Jang TR, Wu CL, Chang CM, Hung W, Fang SH, Chang CK. Effects of carbohydrate, branched-chain amino acids, and arginine in recovery period on the subsequent performance in wrestlers. Journal of the International Society of Sports Nutrition. 2011; 8:21.

12) Knechtle B, Mrazek C, Wirth A, Knechtle P, Rust CA, Senn O, Rosemann T, et al. Branched-chain amino acid supplementation during a 100-km ultra-marathon-a randomized controlled trial. Journal of Nutritional Science and Vitaminology. 2012; 58(1):36-44.

13) Lollo PC, Amaya-Farfan J, de Carvalho-Silva LB. Physiological and physical effects of different milk protein supplements in elite soccer players. Journal of Human Kinetics. 2011; 30:49-57.

14) Lowery RP, Joy JM, Dudeck JE, Oliveira de Souza E, McCleary SA, Wells S, et al. Effects of 8 weeks of Xpand "X pre workout supplementation on skeletal muscle hypertrophy, lean body mass, and strength in resistance trained males. Journal of the Internation Society of Sports Nutrition. 2013; 10(1):44

15) Lustgarten MS, Price LL, Chale A, Phillips EM, Fielding RA. Branched chain amino acids are associated with muscle mass in functionally limited older adults. The Journals of Gerontology. Series A Biological Sciences and Medical Sciences. 2013.

16) Ra SG, Miyazaki T, Ishikura K, Nagayama H, Komine S, et al. Combined effect of branched-chain amino acids and taurine supplementation on delayed onset muscle soreness and muscle damage in high-intensity eccentric exercise. Journal of the International Society of Sports Nutrition. 2013; 10(1):51

17) Rodwell VW. Chapter 29. Catabolism of the Carbon Skeletons of Amino Acids. In: Murray RK, Bender DA, Botham KM, Kennelly PJ, Rodwell VW, Weil P. eds. Harper's Illustrated Biochemistry, 29e. New York: McGrawHill; 2012.

18) Rodwell VW. Chapter 28. Catabolism of Proteins \& of Amino Acid Nitrogen. In: Murray RK, Bender DA, Botham KM, Kennelly PJ, Rodwell VW, Weil P. eds. Harper's Illustrated Biochemistry, 29e. New York: McGraw-Hill; 2012.

19) Sharp CP, Pearson DR. Amino acid supplements and recovery from high-intensity resistance training. Journal of Strength and Conditioning Research. 2010; 24(4):1125-30.

20) Wisnik P, Chmura J, Ziemba AW, Mikulski T, Nazar K. The effect of branched chain amino acids on psychomotor performance during treadmill exercise of changing intensity simulating a soccer game. Applied Physiology, Nutrition, and Metabolism. 2011; 36(6):856-62. 